\title{
THz nanoscopy of plasmonic resonances with a quantum cascade laser
}

\author{
Riccardo Degl'Innocenti ${ }^{1,}$, Robert Wallis ${ }^{1,}$, , Binbin Wei ${ }^{1}$, Long Xiao ${ }^{1,2}$, Stephen J. Kindness ${ }^{1}$, Oleg \\ Mitrofanov $^{3}$, Philipp Braeuninger-Weimer ${ }^{2}$, Stephan Hofmann ${ }^{2}$, Harvey E. Beere ${ }^{1}$, David A. Ritchie ${ }^{1}$ \\ ${ }^{1}$ Cavendish Laboratory, University of Cambridge, J. J Thomson Avenue, CB3 OHE Cambridge, United Kingdom \\ ${ }^{2}$ Department of Engineering, University of Cambridge, J. J. Thomson Avenue, CB3 OFA Cambridge, United Kingdom \\ ${ }^{3}$ Department of Electronic and Electrical Engineering, University College London, Torrington Place, WC1E 7JE London, United Kingdom \\ ${ }^{\S}$ These authors equally contributed
}

\begin{abstract}
We present a THz scattering near field optical microscope (s-SNOM) based on a quantum cascade laser implemented both as source and detector in a self-mixing scheme utilizing resonant quartz tuning forks as a sensitive nano-positioning element. The home-made s-SNOM, based on a resonant tuning fork and metallic tip, operates in tapping mode with a spatial resolution of $\sim 78 \mathrm{~nm}$. The quantum cascade laser is realized from a bound-to-continuum active region design with a central emission of $\sim 2.85 \mathrm{THz}$, which has been lens-coupled in order to maximize the feedback into the laser cavity. Accordingly, the spatial resolution corresponds to $>\lambda / 1000$. The s-SNOM has been used to investigate a bi-dimensional plasmonic photonic crystal and to observe the plasmonic resonances supported by coupled plasmonic planar antennas, showing remarkable agreement with the theoretical predictions. The compactness, unique sensitivity and fast acquisition capability of this approach makes the proposed sSNOM a unique tool for solid-state investigations and biomedical imaging.
\end{abstract}

Keywords: Near-field microscopy, terahertz, plasmonics, photonic crystals, quantum cascade laser, self-mixing detection

The terahertz (THz) frequency range, $0.1-10 \mathrm{THz}$, lies in the millimetre and sub-millimetre wavelength range of the electromagnetic spectrum and represents a fast-evolving research area. The increasing efforts in $\mathrm{THz}$ science and technology are driven by many fields where such radiation finds applications, mainly in communications, spectroscopy and imaging [1,2]. In particular, $\mathrm{THz}$ imaging is appealing in several areas. In biomedical research field this non-ionizing radiation has been used for diagnostics, due to its capability of discriminating between healthy and cancerous tissues [3]. Near-field imaging techniques have great potential in many applications, ranging from the investigation of the optical properties of solid-state and two-dimensional materials [4-6] to the excitation and direct retrieval of plasmonic resonant modes [8-11]. In semiconductor physics, $\mathrm{THz}$ radiation has proved to be a viable tool for inspection of integrated devices with sub-wavelength resolution [7]. It is well known that $\mathrm{THz}$ radiation is sensitive to the doping level of semiconductor devices. Accordingly, it can be implemented for the inspection and mapping of quantum objects even when covered by a dielectric layer, as an alternative and/or complementary technique to photoluminescence techniques. The most commonly used scattering near-field systems are based on modified atomic force microscopes or commercially available instruments, and have proved themselves as efficient tools for the direct mapping and excitation of resonant plasmonic modes in the mid-infrared spectral range [8-10]. Bi-dimensional materials such as topological insulators [12] or graphene [13] are known to also support surface plasmons in the $\mathrm{THz}$ range. Graphene has also been proposed as the basic building block of integrated circuitry [14]. However, the direct excitation of surface plasmons is hindered by the wavevector mismatch between the incoming radiation and the supported modes, and typically requires direct patterning or prism-coupling schemes. Near-field microscope probes directly interact with the surface of the object of study through evanescent waves, thus naturally overcoming the dispersion difference. Near field microscopy thus provides a valuable tool for the excitation of all-plasmonic or hybrid devices. The design of future THz integrated optoelectronic devices, such as amplitude [15-17], frequency [18] and polarization [19] modulators, or detectors [20-22], based on metamaterial/plasmonic subwavelength resonances, relies on the detailed analysis of the properties of a single unit, rather than on ensemble averages, thus enforcing the need for a subwavelength inspection tool. Near-field microscopes operating in the THz normally implement cumbersome and unstable gas lasers as sources, such as a $\mathrm{CH}_{3} \mathrm{OH}$ laser pumped by a $\mathrm{CO}_{2}$ laser [7,11]. Quantum cascade lasers (QCLs) [23] are a more compact, stable and 
versatile source to be used in these arrangements. The use of QCLs in aperture $[24,25]$ and apertureless [26] scanning near field optical microscopes has already been reported with a resolution $>1 \mu \mathrm{m}$ as a proof of principle, without an efficient tip/surface feedback system. The self-mixing scheme represents an elegant and powerful approach to $\mathrm{THz}$ detection [27-29]. Instead of using slow-responding and/or cryogenic devices such as pyroelectric or Si-bolometer for detection, self-mixing is based on the perturbation introduced by the radiation scattered into the laser cavity by the tip/surface system. By monitoring the change in bias applied to the QCL, or the QCL power, it is possible to retrieve information about the amplitude and phase of the scattered electric field. This approach presents many advantages over conventional techniques. It allows a more compact scheme, by eliminating the need for extra components. It is an extremely sensitive detection scheme which has been reported to be sensitive to $\mathrm{nW}$ power levels [27], thus making it extremely suitable for the detection of intrinsically weak, omnidirectional scattered signals. Finally, it is a fast detection scheme [29], since the ultimate limit is given by the QCL laser dynamics, which typically take place on time scales of picoseconds. The quartz resonant tuning forks (QTF) have been implemented in near field microscopy as an alternative to the atomic force microscope cantilevers [30-32]. The resonances supported by these economic and compact elements are based on the piezoelectric effect. Accordingly, a mechanical modification of the resonance amplitude, frequency or phase translates into a current/voltage change which then provides the feedback mechanism. Because of their high Q-factor and superior stiffness compared to cantilevers, the QTFs are implemented together with sharp metallic tips as an efficient sensitive element in near-field microscopy for the achievement of a constant distance between tip and surface. Their simple circuitry and the lack of an optical illumination feedback system, as required by the cantilever approach, make them the most favourable choice in cryogenic environments $[33,34]$ or in imaging systems susceptible to being optically perturbed.

Results and Discussion: Here, we report a THz s-SNOM system with a resolution of $\sim 78 \mathrm{~nm}$, based on a self-mixing detection scheme and implementing QCLs emitting around $2.85 \mathrm{THz}$ as the source /detector. This home-made system has been used to image the plasmonic resonances supported by a bidimensional photonic crystal triangular hole array [35] and the electric field enhancement supported in the gap of a graphene/plasmonic antenna THz detector [21].

The QCL fabrication and coupling to a Silicon lens is described in the Methods section. A schematic of the experimental setup is showed in Fig. 1 a). The laser light is collimated and focused by two 2 inch $\mathrm{f} / 1$ off-axis parabolic mirrors with $\sim 30^{\circ}$ incident angle. When the beam is illuminating the metallic tip, a positive change in the laser power is detected. The apparatus is then arranged for the collection of the scattered light. The self-mixing scheme used for the acquisition of the $\mathrm{THz}$ scattered light is reported in Fig. 1 b). To amplify the small voltage perturbation on the laser bias $\mathrm{V}_{\mathrm{QCL}}$ caused by the scattered light $\mathrm{V}_{\text {scatt }}$, it is first mixed with the bias of an identical pulser $\mathrm{V}_{\text {Ref }}$ synchronized to the repetition rate of the QCL $f_{Q C L}$, kept fixed to $2 \mathrm{kHz}$. The voltage difference between the two pulsers $\mathrm{V}_{1}$ and $\mathrm{V}_{2}$ in Fig. 1 a) was kept as low as possible in order to use the minimum sensitivity scale on the lock-in amplifier, thus minimizing the noise. The voltage difference has been magnified in Fig. 1 b) for clarity. The difference in voltage is then amplified and fed to the lock-in amplifier which has the resonant frequency of the tuning fork $\mathrm{f}_{\mathrm{tf}}$ as reference. The tuning forks used had a typically resonant frequency of $\sim 30 \mathrm{kHz}$. A higher feedback in the laser cavity corresponds to a reduced bias measured on the QCL, as schematically showed in Fig. 1 b) and experimentally presented in Fig. S1. A metallic tip with radius $<50 \mathrm{~nm}$ is mounted in tapping mode configuration. Further details on the set-up and the data acquisition procedure are reported in the Methods section. A typical measurement example is showed in Fig. 2. This picture reports a) the topography, b) the $\mathrm{THz}$ scattered image and c) the optical micrograph of the sample, which consisted of metallic features over a $\mathrm{SiO}_{2}$ substrate. The sample has two overlapping metallic areas achieved with optical lithography, a vertical stripe (Ti/Au, nominal thickness 10/500 nm) and with an electron beam lithography horizontal stripe (Ti/Au nominal thickness 80/20 nm). According to the standard scattering theory, as presented in [36-38] the tip-surface system is modeled as a spherical particle above a planar surface. The incident light induces a dipole in the tip which is mirrored into the substrate, and the combined dipole-system irradiates with an effective polarizability $\alpha_{\text {eff }}$ given by Eq. 1 : 


$$
\alpha_{e f f}=\frac{\alpha(1+\beta)}{1-\left(\frac{\alpha \beta}{16 \pi(a+z)^{3}}\right)}
$$

Where $\alpha=4 \pi \mathrm{a}^{3}\left(\varepsilon_{\mathrm{tip}}-1\right) /\left(\varepsilon_{\mathrm{tip}}+2\right), \beta=\left(\varepsilon_{\mathrm{sub}}-1\right) /\left(\varepsilon_{\mathrm{sub}}+1\right)$, a is the radius of the tip's apex, $\mathrm{z}$ is the relative distance between sample and substrate and $\varepsilon_{\text {tip }}, \varepsilon_{\text {sub }}$ are the dielectric constant of the tip and substrate, respectively. Assuming a value for the real part of the dielectric constant $\varepsilon_{\text {sub }}$ of 3.9 for the $\mathrm{SiO}_{2}$ and of $-10,0000$ [39,40] for the Au features, an $\varepsilon_{\text {tip }}$ of - 40,000 [41] for the W-tip having an apex radius of $\sim 30 \mathrm{~nm}$, the model predicts a reduced polarizability, hence scattering efficiency, for a dielectric substrate with respect to a metallic one. The THz image presented in Fig. 2 b) shows comparable values for the scattering signals on the $\mathrm{SiO}_{2}$ and the thicker $\mathrm{Au}$ evaporation. According to the polarizability values calculated using Eq. 1 and reported in Fig. S2 in the SI, the ratio between the polarizability, and hence the scattering, yielded by the two different surfaces is $\sim 1.3$. The $\mathrm{THz}$ signal recorded over the metallic evaporation obtained via ebeam lithograpy, corresponding to the horizontal stripe, presents a significantly increased signal in comparison with the thick metal evaporation area and the $\mathrm{SiO}_{2}$ area. This is attributed to the increased roughness, as also shown by the topography, and uniformity of this area. The induced dipole itself and the tip radius have sizes comparable to the roughness and to the total thickness of this metallic layer which might interact efficiently with the total scattering.

A second scan over the bottom area of Fig. 2 has been recorded with a $100 \mathrm{~nm}$ step-size and the topography and corresponding THz signal are reported in Fig. 3 a) and b), respectively. Several profiles have been extracted from the dashed area of Fig. 3 b), to measure the resolution, which, as shown in Fig. $3 \mathrm{c}$ ), was calculated to be $78 \mathrm{~nm}$, by using the 10\%-90\% criterion. This corresponds to a spatial resolution of $\sim \lambda / 1200$, which is comparable to the resolution achieved at these frequencies with a methanol gas laser [7], but obtained with an emitting source with < mW emitting power and without the need of an extra detecting element. At the same time, this system yielded an improvement of more than 10 times compared to similar near-field systems implementing THz QCLs [24,25].

The THz s-SNOM system was then used for mapping the plasmonic resonances supported by resonant metallic features. A surface emitting photonic crystal (SEPHC) QCL [35] based on triangular arrays of holes and emitting around $2.85 \mathrm{THz}$ was chosen since its emission overlapped well with the s-SNOM frequency. The interest in this sample, which was used as a passive element at room temperature, stems from its patterned metallic top contact area, and the relative plasmonic resonances supported. A scanning electron microscope (SEM) micrograph of the top contact of the SEPHC is shown in Fig. 4 a). The patterned metallic surface of the QCL supports dipole modes, as shown in the normalized E-field reported in Fig. 4 b) simulated with the Comsol Multiphysics finite element commercial software following a procedure already explained in [16-17]. The simulated resonant plasmonic modes supported at $2.8 \mathrm{THz}$ have two distinct peaks along the direction of the incident E-field. Fig. $4 \mathrm{c}$ ) and d) report the topography and the retrieved $\mathrm{THz}$ scattered signal acquired simultaneously together with the respective profiles extracted along the two corresponding lines. The topography is consistent with the SEM picture and the profiles acquired along the two directions, showing the $\sim 0.6 \mu \mathrm{m}$ thick metal triangular lattice of holes along the two directions. The image has been smoothed over 3 points and in some points the tip lost contact with the surface, resulting in missing rows, which have been substituted with the first neighbouring points. This operation did not affect the $\mathrm{THz}$ signal and also had marginal effects on the topography, as shown by the original data reported in the additional data folder. Because of the lift off the borders of the holes opened on the metallic surface present significant sharp ripples, as showed by the SEM of the inset in Fig. 4 a) and correctly recorded by the topography in Fig. 4 c). The THz image and the corresponding profiles, shown in Fig. $4 \mathrm{~d}$ ), in contrast to the topography, exhibit an asymmetry in the vertical direction with respect to the horizontal one. In particular, two annular peaks are evident along the polarization direction, in good agreement with the simulations presented in Fig. 4 b). This is consistent with the resonant plasmonic modes supported by the photonic crystal metallic structure. However, due to the sharp metallic rim in the fabrication of the triangular hole array, the THz scattered signal is also strongly influenced by the scanning direction. This can provide an additional signal which is likely to be the predominant effect for this specific sample. Because of the irregular metallic border, an increase in the vertical movement of the tip during the scan translates into an increased proximity of the tip and consequently a stronger scattering signal compared to smoother scanning paths, as shown in 
the SI in Fig. S2. This uniquely sensitive approach also allows the retrieval of dielectric/plasmonic modes supported in buried resonant structures at these frequencies, such as the one shown in Fig. 5.

A plasmonic antenna array loaded with graphene was chosen as the object of investigation, similar to the devices already investigated for the bolometric detection of THz QCLs in [21], but having a resonant frequency centered around $2.9 \mathrm{THz}$. The single unit element of the array is composed of planar metallic antennas of size $15 \times 2 \mu \mathrm{m}^{2}$, fabricated on top of a $\mathrm{SiO}_{2} / \mathrm{Si}$ substrate and shorted by graphene regions with $3 \mu \mathrm{m}^{2}$ area. All the resonant elements are encapsulated in an $\mathrm{Al}_{2} \mathrm{O}_{3}$ dielectric film up to $100 \mathrm{~nm}$ thick deposited by atomic layer deposition. In contrast to the previous device, this presents a smoother metallic border, and the encapsulation with the dielectric layer of $\mathrm{Al}_{2} \mathrm{O}_{3}$ removed the possibility of any other source of extra scattering. The gap between the two antenna arms is $\sim 300 \mathrm{~nm}$, as shown by the topography image acquired with the s-SNOM, and the extracted profiles of Fig. 5 a) and b), respectively. When the orientation of the incident polarization was along the main axis of the antennas, the $\mathrm{THz}$ topography and the corresponding extracted profile, shown in Fig. 5 c) and d) respectively, presented a strong E-field enhancement in the gap area. This is in very good agreement with the simulation obtained for the normalized E-field by using Comsol Multiphysics software and reported in the inset of Fig. $5 \mathrm{~d}$ ). Conversely, when the polarization is perpendicular to the main axis of the antenna, a weak $\mathrm{THz}$ signal is still observable at the boundary between the metallic antennas and the dielectric, as shown in the SI, but no resonant enhancement of the E-field is observed in the gap. It seems from the pictures acquired in Fig. 4 and Fig. 5 that the system is capable of retrieveing the enhancement of the E-field supported by resonant structures. Since the s-SNOM was operating in tapping mode, it should be more sensitive to the vertical component of the E-field rather than to the in-plane component. According to the simulation performed with Comsol multiphysics the two components should have comparable strength. In the case on the resonant antenna of Fig. 5, we have not observed a dipolar mode corresponding to the vertical component of the E-field, but this was attributed to the limited resolution of the system. Operating the s-SNOM in shear-force mode rather than tapping mode might help in determining which component is predominantly retrieved by this system. Demodulation at higher harmonics, which is a common strategy to discriminate the contribution arising from the polarized tip and the illuminated substrate, did not yield a significant improvement in these experiments, but mainly reduced the total collected signal. This effect can be attributed to the limited frequency response of the electronics implemented for demodulating the signal, or to the self-mixing detection scheme itself, which is a strongly nonlinear process based on the amount of scattered photons fed back into the laser cavity.

By further reducing all sources of noise, atmospheric perturbations, and placing the whole apparatus in a nitrogen purged environment, the resolution is expected to improve to $<30 \mathrm{~nm}$, a level capable of investigating low-dimensional objects, such as quantum dots or nanowires [42]. The main limits of the current system are represented by the non-optimal isolation from vibrations, and by the environment perturbations. The first issue prevents the system from reaching its full capability in resolution, while the latter is instead responsible for the unwanted fluctuations in the reflected $\mathrm{THz}$ signal due to the strong water absorption in the laser path.

This home-made s-SNOM system has advantages over modified atomic force microscopes, in terms of compactness and versatility, thus presenting the possibility of use at cryogenic temperatures. This would facilitate completely new scenarios, allowing the active manipulation of the quantum properties of low-dimensional quantum objects, such as nanowires and quantum dots (QDs). We aim to locally excite QDs or quantum molecules, by selectively injecting THz photons in a photon-assisted tunnelling experiment where transport is enabled by intraband absorption in the confined hole states of QDs, or in the electronic band for molecules. A plethora of new experiments can then be pursued aiming to perform spectroscopy of confined electron-hole systems. Finally, the selective injection of single $\mathrm{THz}$ photons could be used to trigger the emission of a single near-infrared photon from a $\mathrm{QD}$, thus providing a THz-to-Telecom bridge in a quantum communication system, and leading to the realization of an integrated quantum photon converter. In conclusion, we have demonstrated a THz s-SNOM based on the self-mixing technique using QCLs. A $78 \mathrm{~nm}$ resolution, corresponding to > $\lambda / 1000$, was achieved with a QCL emitting around $2.85 \mathrm{THz}$. The sensitivitiy of this approach has been further enhanced by partly suppressing lasing action and simultaneously increasing the collection efficiency with an antireflection coated lens attached to a laser facet. The system has been successfully implemented to retrieve the $\mathrm{THz}$ image of a photonic crystal lattice of triangular holes and to map the 
plasmonic modes supported by planar antennas loaded with graphene. This represents significant progress in the field of $\mathrm{THz}$ microscopy, and paves the way to unique investigations of semiconductor quantum objects, bidimensional materials and biological samples.

\section{Methods}

QCL fabrication: the QCL was fabricated with a single plasmon waveguide, with a bound-tocontinuum active region emitting around $2.85 \mathrm{THz}$. In order to enhance the sensitivity of the system, as well as the collection of the scattered light, a silicon lens was attached to the QCL [43] with an $18.5 \mu \mathrm{m}$ thick antireflection parylene coating. The lens mounting had two primary aims; increasing the collection efficiency of the back-scattered light, and increasing the cavity mirror loss, rendering the device more similar to a quantum cascade amplifier [44]. By carefully attaching the lens to the facet of the laser using a layer of PMMA (polymethyl methacrylate), the emission was strongly reduced but not fully suppressed. A partially suppressed laser emission is needed in order to align the beam onto the metallic tip. The voltage-light-current characteristics of the device with and without feedback are presented in the SI. The laser was operated at the current density corresponding to the maximum emitted power. The optical path was finely adjusted in order to have a maximal back-scattered signal into the cavity.

Acquisition procedure: the laser was mounted into the cryostat such that the E-field had a polarization component along the tip shaft. The tuning fork with the metallic tip was mounted on a printed circuit board connected with a piezoelectric motor stage arranged in the vertical z direction with $65 \mu \mathrm{m}$ travel range and $0.13 \mathrm{~nm}$ minimum step-size. When the system is brought into close proximity to the sample surface, the tuning fork resonance is damped and the frequency, phase or Q-factor can be used to close the feedback loop and keep a constant tip/sample distance, typically between $30 \mathrm{~nm}$ and $100 \mathrm{~nm}$. The sample is then scanned in the xy plane by using two piezoelectric stages with $250 \mu \mathrm{m}$ travel range in each direction and $0.4 \mathrm{~nm}$ minimum step size. For each xy position, measurements were recorded for both the height of the tip, yielding the surface topography, and the signal from the lock-in amplifier, corresponding to the $\mathrm{THz}$ scattered signal.

\section{Acknowledgements}

RDI, HEB, OM and DAR acknowledge financial support from the Engineering and Physical Sciences Research Council (Grant No. EP/J017671/1, Coherent Terahertz Systems and Grant No. EP/P021859/1 HyperTerahertz - High precision terahertz spectroscopy and microscopy). SH. and PAB. acknowledge financial support from the Engineering and Physical Sciences Research Council (Grant No. EP/K016636/1, GRAPHTED).

\section{Author Information}

Corresponding author.*Email: $r d 448 @$ cam.ac.uk. ORCID ID;

Riccardo Degl'Innocenti: 0000-0003-2655-1997

\section{Supporting Information Available}

Light-current-voltage characteristics of the QCL used in this work with and without the feedback. Effective polarizability calculated for different substrates. Topography and corresponding THz image of the antenna device shown in the main text but with the incident E-field polarization perpendicular to the axis of the antenna. Relative profiles extracted from the topography and the $\mathrm{THz}$ image.

Additional Data: Additional data sets related to this publication are available from the Cambridge University data repository at XXXX.

\section{References}

(1) Dhillon, S. S.; Vitiello, M. S.; Linfield, E. H.; Davies, A. G.; Hoffmann, M. C.; Booske, J.; Paoloni, C.; Gensch, M.; Weightman, P.; Williams, G. P.; Castro-Camus, E.; Cumming, D. R. S.; Simoens, F.; Escorcia-Carranza, I.; Grant, J.; Lucyszyn, S.; Kuwata-Gonokami, M.; Konishi, K.; Koch, M.; Schmuttenmaer, C. A.; Cocker, T. L.; Huber, R.; Markelz, A. G.; Taylor, Z. D.; Wallace, V. P.; 
Axel Zeitler, J.; Sibik, J.; Korter, T. M.; Ellison, B.; Rea, S.; Goldsmith, P.; Cooper, K. B.; Appleby, R.; Pardo, D.; Huggard, P. G.; Krozer, V.; Shams, H.; Fice, M.; Renaud, C.; Seeds, A.; Stöhr, A.; Naftaly, M.; Ridler, N.; Clarke, R.; Cunningham, J. E.; Johnston, M. B. The 2017 terahertz science and technology roadmap J. Phys. D: Appl. Phys. 2017, 50, 043001.

(2) Lee, Y. S. Principles of Terahertz Science and Technology; Springer-Verlag USA, 2009.

(3) Cheon, H.; Yang, H.-J.; Lee, S.-H.; Kim, Y. A.; Son, J.-H. Terahertz molecular resonance of cancer DNA. Sci. Rep. 2016, 6, 37103.

(4) Yazyev, O. V.; Chen, Y. P. Polycrystalline graphene and other two-dimensional materials. Nat. Nanotech. 2014, 9, 755-767.

(5) Buron J. D.; Petersen, D. H.; Boeggild, P.; Cooke, D. G.; Hilke, M.; Sun, J.; Whiteway, E.; Nielsen, P. F.; Hansen, O.; Yurgens, A.; Jepsen, P. U. Graphene Conductance Uniformity Mapping. Nano Lett. 2012, 12, 5074-5081.

(6) Milot, R. L.; Sutton, R. J.; Eperon, G. E.; Haghighirad, A. A.; Hardigree, J. M.; Miranda, L.; Snaith, H. J.; Johnston, M. B.; Herz, L. M. Charge-Carrier Dynamics in 2D Hybrid Metal-Halide Perovskites. Nano Lett. 2016, 16, 7001-7007.

(7) Huber, A. J.; Keilmann, F.; Wittborn, J.; Aizpurua, J.; Hillenbrand, R. Terahertz Near-Field Nanoscopy of Mobile Carriers in Single Semiconductor Nanodevices. Nano Lett. 2008, 8, 37663770.

(8) Schnell, M.; Sarriurgarte, P.; Neuman, T.; Khanikaev, A. B.; Shvets, G.; Aizpurua, J.; Hillenbrand, R. Real-Space Mapping of the Chiral Near-Field Distributions in Spiral Antennas and Planar Metasurfaces. Nano Lett. 2016, 16, 663-670.

(9) Chen, J.; Badioli, M.; Alonso-Gonzalez, P.; Thongrattanasiri, S.; Huth, F.; Osmond, J.; Spasenovic, M.; Centeno, A.; Pesquera, A.; Godignon, P.; Zurutuza Elorza, A.; Camara, N.; Garcia de Abajo, F. J.; Hillenbrand, R.; Koppens, F. H. L. Optical nano-imaging of gate-tunable graphene plasmons. Nature 2012, 487, 77-81.

(10) Nikitin, A. Y.; Alonso-Gonzalez, P.; Velez, S.; Mastel, S.; Centeno, A.; Pesquera, A.; Zurutuza, A.; Casanova, F.; Hueso, L. E.; Koppens, F. H. L.; Hillenbrand, R. Real-space mapping of tailored sheet and edge plasmons in graphene nanoresonators. Nat. Photon. 2016, 10, 239-244.

(11) Alonso-Gonzalez, P.; Nikitin, A. Y.; Gao, Y.; Woessner, A.; Lundeberg, M. B.; Principi, A.; Forcellini, N.; Yan, W.; Velez, S.; Huber, A. J.; Watanabe, K.; Taniguchi, T.; Casanova, F.; Hueso, L. E.; Polini, M.; Hone, J.; Koppens, F. H. L.; Hillenbrand, R. Acoustic terahertz graphene plasmons revealed by photocurrent nanoscopy. Nat. Nanotech. 2017, 12, 31-35.

(12) Di Pietro, P.; Ortolani, M.; Limaj, O.; Di Gaspare, A.; Giliberti, V.; Giorgianni, F.; Brahlek, M.; Bansali, N.; Koirala, N.; Oh, S.; Calvani, P.; Lupi, S. Observation of Dirac plasmons in a topological insulator. Nat. Nanotech. 2013, 8, 556-560.

(13) Ju, L.; Geng, B.; Horng, J.; Girit, C.; Martin, M.; Hao, Z.; Bechtel, H. A.; Lian, X.; Zettl, A.; Ron Shen, Y.; Wang, F. Graphene plasmonics for tunable terahertz metamaterials. Nat. Nanotech. 2011, 6, 630-634.

(14) Vakil, A.; Engheta, N. Transformation Optics Using Graphene. Science 2011, 332, 1291-1294.

(15) Jadidi, M. M.; Sushkov, A. B.; Myers-Ward, R. L.; Boyd, A. K.; Daniels, K. M.; Gaskill, D. K.; Fuhrer, M. S.; Drew, H. D.; Murphy, T. E. Tunable Terahertz Hybrid Metal-Graphene Plasmons. Nano Lett. 2015, 15, 7099-7104.

(16) Jessop, D. S.; Kindness, S. J.; Xiao, L.; Braeuninger-Weimer, P.; Lin, H.; Ren, Y.; Ren, C. X.; Hofmann, S.; Zeitler, J. A.; Beere, H. E.; Ritchie, D. A.; Degl'Innocenti, R. Graphene based plasmonic terahertz amplitude modulator operating above $100 \mathrm{MHz}$. Appl. Phys Lett. 2016, 108, 171101.

(17) Degl'Innocenti, R.; Jessop, D. S.; Sol, C. W. O.; Xiao, L.; Kindness, S. J.; Lin, H.; Zeitler, J. A.; Braeuninger-Weimer, P.; Hofmann, S.; Ren, Y.; Kamboj, V. S.; Griffiths, J.; Beere, H. E.; Ritchie, D. A. Fast Modulation of Terahertz Quantum Cascade Lasers Using Graphene Loaded Plasmonic Antennas. ACS Photon. 2016, 3, 464-470.

(18) Chen, H.-T.; O'Hara, J. F.; Azad, A. K.; Taylor, A. J.; Averitt, R. D.; Shrekenhamer, D. B.; Padilla, W. J. Experimental demonstration of frequency-agile terahertz metamaterials. Nat. Photon. 2008, 2, 295-298. 
(19) Zhou, J.; Chowdhury, D. R.; Zhao, R.; Azad, A. K.; Chen, H.-T.; Soukoulis, C. M.; Taylor, A. J.; O'Hara, J. F. Terahertz chiral metamaterials with giant and dynamically tunable optical activity. Phys. Rev. B 2012, 86, 035448.

(20) Cai, X.; Sushkov, A. B.; Suess, R. J.; Jadidi, M. M.; Jenkins, G. S.; Nyakiti, L. O.; Myers-Ward, R. L.; Li, S.; Yan, J.; Gaskill, D. K.; Murphy, T. E.; Drew, H. D.; Fuhrer, M. S. Sensitive roomtemperature terahertz detection via the photothermoelectric effect in graphene. Nat. Nanotech. 2014, $9,814-819$.

(21) Degl'Innocenti, R.; Xiao, L.; Kindness, S. J.; Kamboj, V.; Wei, B.; Braeuninger-Weimer, P.; Nakanishi, K.; Aria, A.; Hofmann, S.; Beere, H. E.; Ritchie, D. A. Bolometric detection of terahertz quantum cascade laser radiation with graphene-plasmonic antenna arrays. J. Phys. D: Appl. Phys. 2017, 50, 174001.

(22) Degl'Innocenti, R.; Xiao, L.; Jessop, D. S.; Kindness, S. J.; Ren, Y.; Lin, H.; Zeitler, J. A.; Alexander-Webber, J. A.; Joyce, H. J.; Braeuninger-Weimer, P.; Hofmann, S.; Beere, H. E.; Ritchie, D. A. Fast Room-Temperature Detection of Terahertz Quantum Cascade Lasers with GrapheneLoaded Bow-Tie Plasmonic Antenna Arrays. ACS Photon. 2016, 3, 1747-1753.

(23) Williams, B. Terahertz quantum-cascade lasers. Nat. Photon. 2007, 1, $517-525$.

(24) Degl'Innocenti, R.; Montinaro, M.; Xu, J.; Piazza, V.; Pingue, P.; Tredicucci, A.; Beltram, F.; Beere, H. E.; Ritchie, D. A. Differential near-field scanning optical microscopy with THz quantum cascade laser sources. Opt. Express 2009, 26, 23785-23792.

(25) Mitrofanov, O.; Viti, L.; Dardanis, E.; Caterina Giordano, M.; Ercolani, D.; Politano, A.; Sorba, L.; Vitiello, M. S. Near-field terahertz probes with room-temperature nanodetectors for subwavelength resolution imaging. Sci Rep. 2017, 7, 44240.

(26) Dean, P.; Mitrofanov, O.; Keeley, J.; Kundu, I.; Li, L.; Linfield, E. H.; Davies, A. G. Apertureless near-field terahertz imaging using the self-mixing effect in a quantum cascade laser Appl. Phys. Lett. 2016, 108, 091113.

(27) Dean, P.; Lim, Y. L.; Valavanis, A.; Kliese, R.; Nikolić, M.; Khanna, S. P.; Lachab, M.; Indjin, D.; Ikonić, Z.; Harrison, P.; Rakić, A. D.; Linfield, E. H.; Davies, A. G. Terahertz imaging through selfmixing in a quantum cascade laser. Opt. Lett. 2011, 36, 2587-2589.

(28) Valavanis, A.; Dean, P.; Lim, Y. L.; Alhathlool, R.; Nikolic, M.; Kliese, R.; Khanna, S. P.; Indjin, D.; Wilson, S. J.; Rakic, A. D.; Linfield, E. H.; Davies, A. G. Self-Mixing Interferometry With Terahertz Quantum Cascade Lasers. IEEE Sensors J. 2013, 13, 37-43.

(29) Ren, Y.; Wallis, R.; Jessop, D. S.; Degl'Innocenti, R.; Klimont, A.; Beere, H. E.; Ritchie, D. A. Fast terahertz imaging using a quantum cascade amplifier. Appl. Phys. Lett. 2015, 107, 011107.

(30) Karrai, K.; Grober, R. Piezoelectric tip-sample distance control for near field optical microscopes. Appl. Phys. Lett. 1995, 66,1842- 1844.

(31) Ruiter, A. G.; Veerman, J. A.; Van Der Werf, K. O.; Van Hulst, N. F. Dynamic behavior of tuning fork shear-force feedback. Appl. Phys. Lett. 1997, 71, 28-30.

(32) Atia, W. A.; Davis, C. C. A Phase-Locked Shear-Force Microscope for Distance Regulation in Near-Field Optical Microscopy. Appl. Phys. Lett. 1997, 70, 405-407.

(33) Rychen, J.; Ihn, T.; Studerus, P.; Herrmann, A.; Ensslin, K. A low-temperature dynamic mode scanning force microscope operating in high magnetic fields Rev. Sci. Instrum. 1999, 70, 2765 2768.

(34) Yang, C. H.; Chang, T. H.; Yang, M. J.; Moore, W. J. A low noise transimpedance amplifier for cryogenically cooled quartz tuning fork force sensors. Rev. Sci. Instrum. 2002, 73, 2713-2716.

(35) Marshall, O. P.; Apostolopoulos, V.; Freeman, J. R.; Rungsawang, R.; Beere, H. E.; Ritchie, D. A. Surface-emitting photonic crystal terahertz quantum cascade lasers. Appl. Phys. Lett. 2008, 93, 171112.

(36) Novotny, L.; Hecht, B. Principle of Nano-Optics; 3rd ed. Cambridge University Press, Cambridge, UK, 2007.

(37) Knoll, B.; Keilmann, F. Near-field probing of vibrational absorption for chemical microscopy Nature 1999, 399, 134-137.

(38) Knoll, B.; Keilmann, F. Enhanced dielectric contrast in scattering-type scanning near-field microscopy. Optics Commun. 2000, 182, 321-328. 
(39) Ordal, M. A.; Long, L. L.; Bell, R. J.; Bell, S. E.; Bell, R. R.; Alexander Jr, R. W.; Ward, C. A. Optical properties of the metals $\mathrm{Al}, \mathrm{Co}, \mathrm{Cu}, \mathrm{Au}, \mathrm{Fe}, \mathrm{Pb}, \mathrm{Ni}, \mathrm{Pd}, \mathrm{Pt}, \mathrm{Ag}, \mathrm{Ti}$, and $\mathrm{W}$ in the infrared and far infrared. Appl. Opt. 1983, 22, 1099-1120.

(40) Ordal, M. A.; Bell, R. J.; Alexander, R. W.; Long, L. L.; Querry, M. R. Optical properties of fourteen metals in the infrared and far infrared: $\mathrm{Al}, \mathrm{Co}, \mathrm{Cu}, \mathrm{Au}, \mathrm{Fe}, \mathrm{Pb}, \mathrm{Mo}, \mathrm{Ni}, \mathrm{Pd}, \mathrm{Pt}, \mathrm{Ag}, \mathrm{Ti}, \mathrm{V}$, and W. Appl. Opt. 1985, 24, 4493-4499.

(41) Ordal, M. A.; Bell, R. J.; Alexander, R. W.; Newquist, L. A.; Querry, M. R. Optical properties of $\mathrm{Al}, \mathrm{Fe}, \mathrm{Ti}, \mathrm{Ta}, \mathrm{W}$, and Mo at submillimeter wavelengths. Appl. Opt. 1988, 27 1203-1209.

(42) Eisele, M.; Cocker, T. L.; Huber, M. A.; Plankl, M.; Viti, L.; Ercolani, D.; Sorba, L.; Vitiello, M. S.; Huber, R. Ultrafast multi-terahertz nano-spectroscopy with sub-cycle temporal resolution. Nat. Photon. 2014, 8, 841-845.

(43) Degl'Innocenti, R.; Shah, Y. D.; Jessop, D. S.; Ren, Y.; Mitrofanov, O.; Beere, H. E.; Ritchie, D. A. Hollow metallic waveguides integrated with terahertz quantum cascade lasers. Opt. Express 2014, 22, 24439-24449.

(44) Ren, Y.; Wallis, R.; Shah, Y. D.; Jessop, D. S.; Degl'Innocenti, R.; Klimont, A.; Kamboj, V.; Beere, H. E.; Ritchie, D. A. Single mode terahertz quantum cascade amplifier. Appl. Phys. Lett. 2014, 105, 141102. 


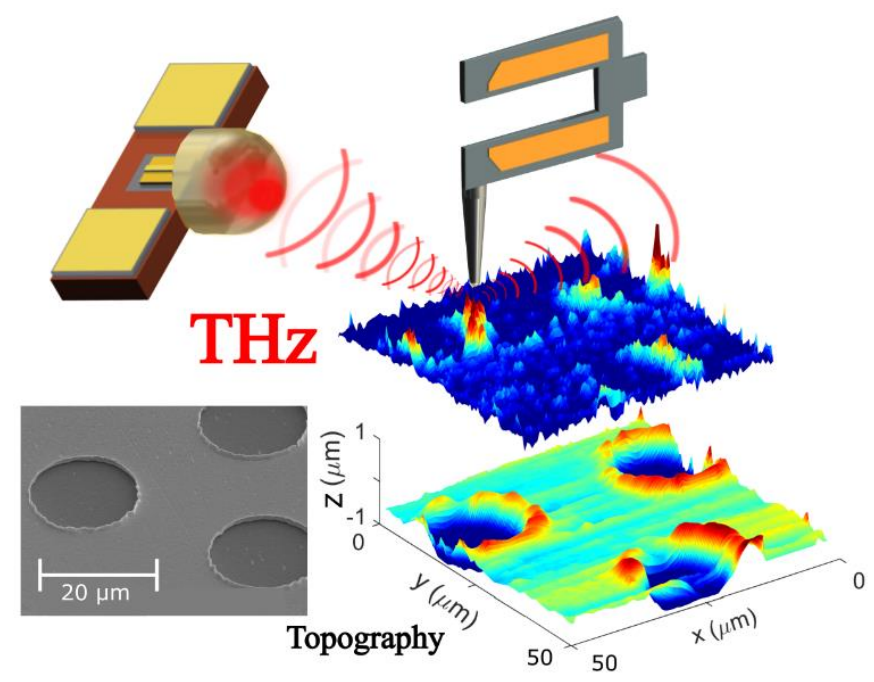

THz nanoscopy of plasmonic resonances with a quantum cascade laser

Riccardo Degl'Innocenti, Robert Wallis, Binbin Wei, Long Xiao, Stephen J. Kindness, Oleg Mitrofanov, Philipp Braeuninger-Weimer, Stephan Hofmann, Harvey E. Beere, David A. Ritchie

This article reports the realization of a near-field scattering optical microscope (s-SNOM) operating in the terahertz frequency range based on resonant quartz tuning forks and on quantum cascade lasers. The laser serves as both source and detecting element in a self-mixing scheme. The microscope achieved a spatial resolution of $>\lambda / 1000$ at the emission frequency of $\sim 2.85 \mathrm{THz}$. The s-SNOM system has been efficiently used to retrieve THz images with $\mathrm{nm}$ resolution of bi-dimensional photonic crystal arrays and resonant plasmonic antennas loaded with graphene, showing itself capable of mapping the electric field enhancement of the supported modes.

This article represents a significant step forward in the physics of sustainability, since it can be readily implemented for the investigation of a plethora of materials and their dielectric and plasmonic properties in the terahertz range with $\mathrm{nm}$ precision, and with a uniquely fast and sensitive detection scheme. Ultimately, this s-SNOM system represents a fundamental tool for the design and study of more efficient functional detectors and modulators based for example on novel materials, such as graphene or other 2D materials. Finally, once operating at cryogenic temperatures, the s-SNOM will have the potential to directly investigate and manipulate low dimensional quantum objects such as quantum dots. Accordingly, it can open new scenarios for the research of fundamental states of matter and of novel quantum circuitry. 